\title{
Tension Pneumocephalus Causing Cerebral Venous Sinus Thrombosis
}

\author{
Nissar Shaikh $^{1 * \#}$, Gulzar Hussain ${ }^{2}$, Adnan Khan ${ }^{3}$, Muhammad Mohsin Khan ${ }^{3}$, Mamdouh Al Mustafa $^{4}$, \\ Moad Ehfeda ${ }^{4}$, Muhammad Zubair ${ }^{2}$ and Umais Momin ${ }^{5}$ \\ ${ }^{1}$ Senior Consultant Surgical Intensive Care, Hamad Medical Corporation, Qatar \\ ${ }^{2}$ Consultant Surgical Intensive Care, Hamad Medical Corporation, Qatar \\ ${ }^{3}$ Department of Neurosurgical Sciences, Hamad Medical Corporation, Qatar
}

${ }^{4}$ Specialist, Department of Anesthesia/ICU\& Perioperative Med. Hamad Medical Corporation, Qatar

${ }^{5}$ Department of Radiology, Hamad Medical Corporation, Qatar

*Corresponding author: Dr. Nissar Shaikh MD, EDIC, Senior Consultant Surgical Intensive Care/Hamad Medical Corporation, Doha-Qatar

\begin{abstract}
Background: Pneumocephalus after craniotomy will absorb within four weeks, but when air causes pressure affects it is a tension pneumocephalus, which may affect consciousness and requires immediate intervention. Tension pneumocephalus causing cerebral sinus thrombosis is not known in the literature. We report a case of tension pneumocephalus causing diabetes insipidus associated with cerebral sinus thrombosis.

Case description: A 38-year-old Asian male had craniotomy with excision of a left frontal mass which extended into the lateral ventricles. External ventricular and subdural drains were inserted at the end of the procedure. $\mathrm{He}$ received mannitol and furosemide intraoperatively. Immediately after surgery he developed tension pneumocephalus, diabetes insipidus and fever. Two days after surgery, magnetic resonance imaging (MRI) showed transverse, sagittal sinus thrombosis extending into the right internal jugular vein. Pre-operative MRI and thrombophilia markers were unremarkable. Cerebrospinal fluid (CSF) analysis did not demonstrate leucocytosis and did not culture bacteria. Anticoagulation was initiated, but on the third day after surgery he developed severe brain swelling and became brain-dead.

Conclusion: In our patient tension pneumocephalus was complicated by diabetes insipidus and leading to cerebral sinus thrombosis after surgery.
\end{abstract}

\section{Keywords}

Tension pneumocephalus, Diabetes insipidus, Dehydration, Craniotomy, Cerebral sinus thrombosis

\section{Introduction}

Pneumocephalus is a common finding after craniotomy [1], but tension pneumocephalus is an acute neurosurgical emergency associated with increased intracranial pressure and neurological deterioration [2]. Criteria for the diagnosis of tension pneumocephalus are: (a) Typical computerised tomography (CT) brain findings (b) Neurological deterioration (c) Hissing sound of escape of air from the skull (d) Immediate improvement in the neurological status upon aspiration of air [3]. In clinical practice it is important to differentiate pneumocephalus from tension pneumocephalus. Tension pneumocephalus requires immediate intervention to decrease the intracranial pressure. Tension pneumocephalus rarely causes diabetes insipidus and never reported to cause fatal cerebral venous sinus thrombosis (CVST). We report a case of tension pneumocephalus causing diabetes insipidus and cerebral sinus thrombosis.

\section{Case Presentation}

A 38-year-old male patient, with no abnormal neurological clinical features and a Glasgow Coma Score (GCS) of 15/15, was admitted with a left frontal tumour extending into the lateral ventricle. The only significant past medical history was diarrhoea four days before admission. Magnetic resonance imaging (MRI) showed normal cerebral sinuses (Figure 1) and haemorrhage

Citation: Shaikh N, Hussain G, Khan A, Khan MM, Al Mustafa M, et al. (2019) Tension Pneumocephalus Causing Cerebral Venous Sinus Thrombosis. Neurosurg Cases Rev 2:023. doi.org/10.23937/26434474/1710023

Accepted: September 11, 2019; Published: September 13, 2019

Copyright: (c) 2019 Shaikh N, et al. This is an open-access article distributed under the terms of the Creative Commons Attribution License, which permits unrestricted use, distribution, and reproduction in any medium, provided the original author and source are credited. 


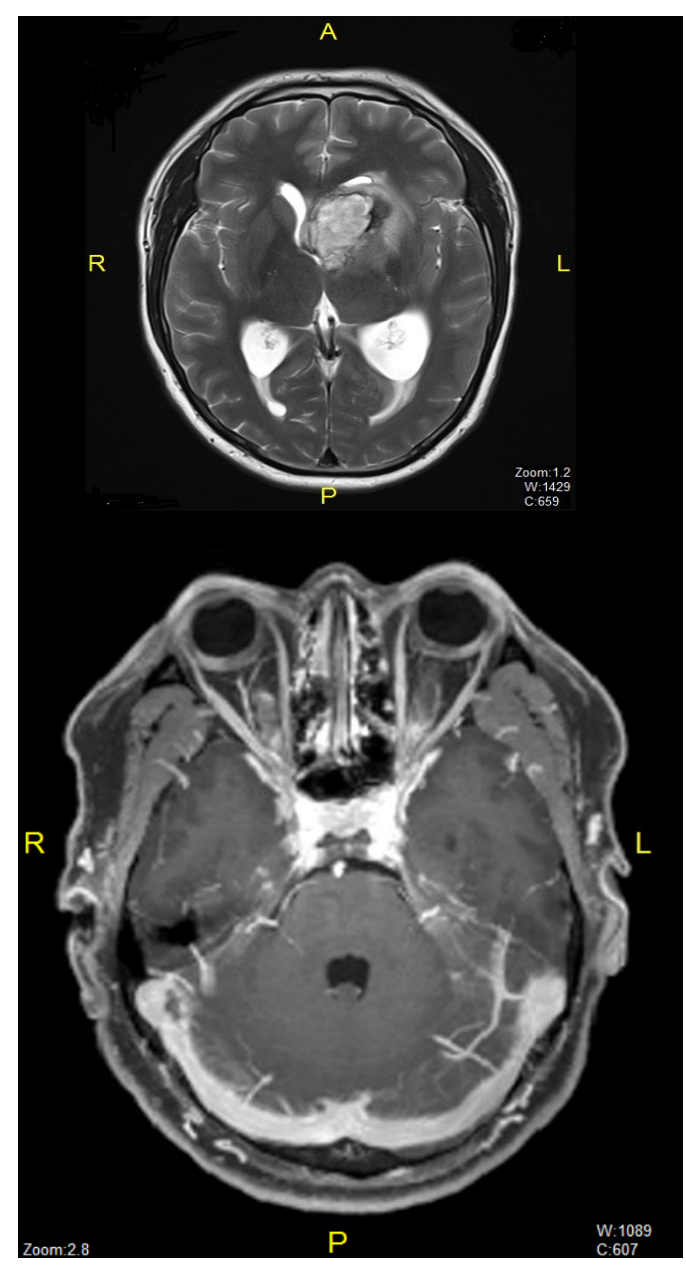

Figure 1: Preoperative MRI showing tumour and Normal sinuses.

within the tumour and surrounding oedema. He was admitted to the surgical intensive care unit (SICU) for observation.

He subsequently underwent craniotomy and excision of tumour in a supine position. External ventricular and subdural drains were inserted at the end of surgery. He received total intravenous anaesthesia with propofol, remifentanil, pancuronium, mannitol, furosemide, phenytoin, and dexamethasone, and was stable hemodynamically throughout. Surgical time was 5 hours. At the end of surgery, return of consciousness was delayed. CT scan of his brain demonstrated tension pneumocephalus (Figure 2). The subdural drain was manipulated and a gush of air obtained. He was kept sedated and ventilated in SICU. He became febrile, $39.2^{\circ} \mathrm{C}$, and pupils were $2 \mathrm{~mm}$ but reacting to light. Temperature was controlled with paracetamol and a cooling device (Arctic Sun ${ }^{\circledR}$, Medivance, Louisville, CO). He remained poyluric for 8 hours, serum sodium was elevated to $164 \mathrm{mmol}^{. \mathrm{l}^{-1}}$ with low urinary specific gravity (1.005). He was diagnosed with diabetes insipidus and received desmopres$\sin 4 \mu \mathrm{g}$ intravenously. He was rehydrated with $0.45 \%$ saline intravenously. His neurological status improved to GCS 10/15(E3M6V1). On the first postoperative day MRI showed right transverse, sagittal sinus and right internal jugular vein thrombosis (Figure 3 ) with complete

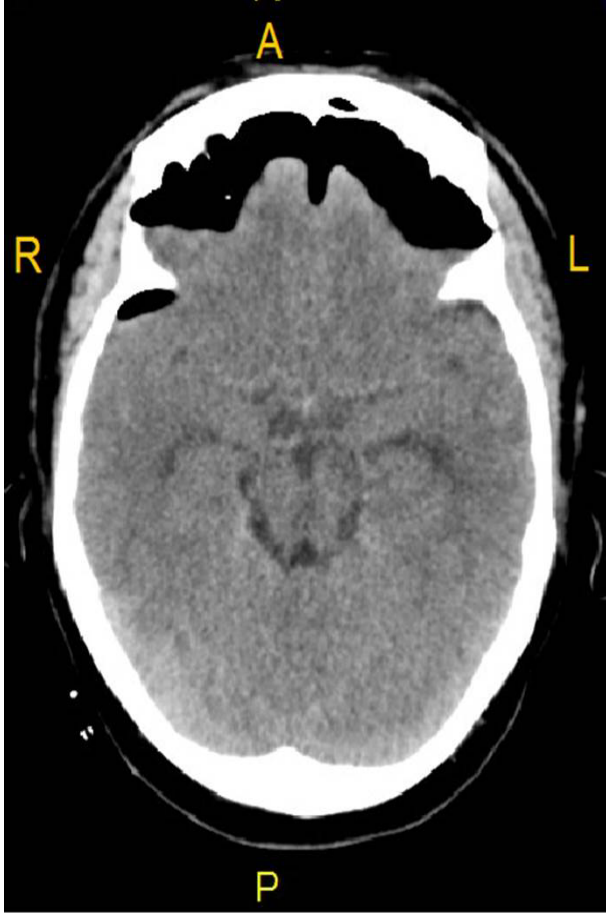

Figure 2: CT brain showing tension pneumocephalus.

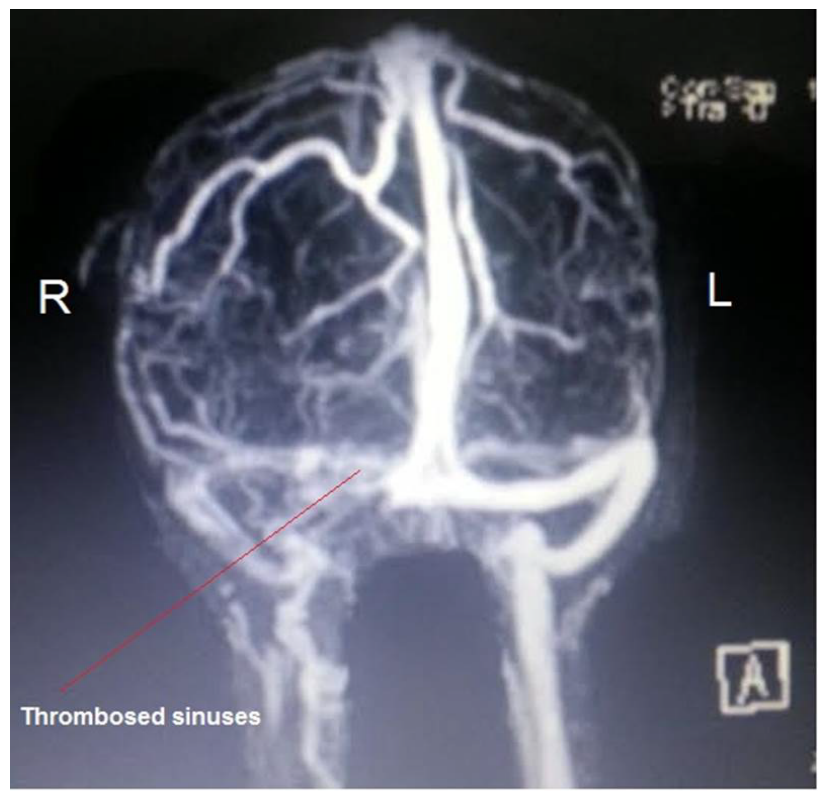

Figure 3: Post-operative MRI showing cerebral sinuses thrombosis.

left sided tumor resection. All thrombophilia markers were negative and cerebro-spinal fluid (CSF) analysis showed no bacterial growth. He was started on heparin anticoagulation. Two days after surgery he was obeying commands and his GCS improved to $11 / 15$. His trachea was extubated after a trial of spontaneous breathing. GCS remained 11/15 after extubation. 8 hours after extubation his extra ventricular drain blocked again. Immediate $\mathrm{CT}$ had demonstrated hydrocephalus, the extra ventricular drain was reinserted, but his pupils became fixed and dilated with absent brainstem reflexes. He was declared brain death on the third day after surgery. 


\section{Discussion}

Pneumocephalus, the presence of air in the cranial cavity without any pressure effects, occurs in most supratentorial craniotomies. It takes up to 4 weeks for complete resolution [4]. When intracranial air causes pressure and decreases the level of consciousness, it is termed tension pneumocephalus. Tension pneumocephalus has a reported incidence of $9 \%$ [5].

The risk factors for tension pneumocephalus are head up position during surgery, long duration of surgery, evacuation of chronic subdural hematoma leaving oxygen in the space, intraoperative use of mannitol and furosemide, use of nitrous oxide during anaesthesia, functioning ventriculo-peritoneal (VP) shunt, CSF drainage and insertion of a drain after the cavity has been irrigated $[1,6]$. Tension pneumocephalus is diagnosed from clinical deterioration and typical CT findings, the Mount Fuji sign, where there is apparent tenting of the brain tissue. Management of tension pneumocephalus is immediate evacuation of air with reduction of intracranial pressure, either by burr-hole or catheter insertion.

Transient diabetes insipidus has been reported in association with tension pneumocephalus [7]. In a series of 22 cases, of pneumocephalus, 2 patients had tension pneumocephalus and both these patient developed transient diabetes insipidus [5].

Apart from diabetes insipidus, our patient had cerebral sinus and internal jugular vein thrombosis with diffuse brain swelling and brain death. This cerebral venous sinus thrombosis was on opposite side of the surgery; hence surgery was not the cause for CVTS. This patient was at risk of developing tension pneumocephalus, because we drain CSF during surgery, and give intra-operative mannitol and furosemide. The combined effects of steroid therapy, tension pneumocephalus, diabetes insipidus, high grade fever with severe dehydration and local brain surgery predisposed the development of cerebral sinus thrombosis extending into the jugular vein. In addition, tension pneumocephalus, by increasing the intracranial pressure, and decreasing venous flow in cerebral veins increased the likelihood of vein thrombosis.

Congenital haemophilia is the most common aeti- ology for cerebral sinus thrombosis (CVST) worldwide, and in Middle Eastern countries Behcet's disease is the most frequent cause [8]. Of patients who develop CVST, $57 \%$ patients will have full recovery [9]. Risk factors for adverse outcome are being male, aged more than 37 years, decreased conscious level, and central nervous system pathology [9].

\section{Conclusion}

Tension pneumocephalus is a rare neurosurgical emergency. In our patient had tension pneumocephalus complicated in to diabetes insipidus and leading to cerebral venous sinuses thrombosis, which was fatal. This is first report of cerebral sinus thrombosis as a complication of tension Pneumocephalus.

\section{References}

1. Zidan I (2012) Pneumocephalus after surgical evacuation of chronic subdural hematoma: Is it a serious complication? Asian J Neurosurg 7: 66-74.

2. Shaikh N, Ummunnisa F, Masood I, Hanssens Y (2010) Tension pneumocephalus as a complication of burr-hole drainage of chronic subdural hematoma: A case report. Surg Neurol Int 1: 27.

3. Sharma BS, Tewari MK, Khosla VK, Pathak A, Kak VK (1989) Tension pneumocephalus following evacuation of chronic subdural haematoma. $\mathrm{Br} \mathrm{J}$ Neurosurg 3: 381-388.

4. Reasoner DK, Todd MM, Scamman FL, Warner DS (1994) The incidence of pneumocephalus after supratentorial craniotomy, observation on the disappearance of intracranial air. Anesthesiology 80: 1008-1012.

5. Yates H, Mark H, Borel CO, Thomas JK (1994) J Neurosurg Anesthesiology 6: 15-20.

6. Sataypathy GC, Dash HH (2000) Tension Pneumocephalus after neurosurgery in supine position. BJA 84: 115-117.

7. Kaplan M, Ozturk MB, Erol FS, Dogukan A, Yakar H (2007) Temporary central diabetes insipidus due to pneumocephalus secondary to posterior fossa surgery: A review of tension pneumocephalus. Neurosurgery Quterly 17: 215.

8. Daif A, Awada A, Al-Rajeh S (1995) Cerebral sinus thrombosis in adult. A study of 40 cases from Saudi Arabia. Stroke 26: 1193-1195.

9. Ferro JM, Canhao P, Stam J, Bousser MG, Baringarre mentana F (2004) Prognosis of cerebral vein and dural sinus thrombosis: Results of international study on cerebral vein and dural sinus thrombosis (ISCVT). Stroke 35: 664670. 